\title{
Effect of a capping layer on the magnetic properties of island nanostructured $\mathrm{Fe}(110)$
}

\author{
J. F. Calleja, ${ }^{\text {a) }}$ M. C. Contreras, and R. Matarranz \\ Departamento de Fisica, Universidad de Oviedo, Facultad de Ciencias, Calvo Sotelo s/n, \\ 33007 Oviedo, Spain \\ E. Navarro \\ Sección Departamental de Fisica Aplicada 1, Facultad de Veterinaria, Universidad Complutense, \\ Avenida Puerta de Hierro s/n, 28040 Madrid, Spain \\ Y. Huttel \\ Instituto de Ciencia de Materiales de Madrid (ICMM-CSIC), 29029 Cantoblanco, Madrid, Spain \\ and Instituto de Microelectrónica de Madrid-IMM (CNM-CSIC), Isaac Newton 8 (PTM) Tres Cantos, \\ 28760 Madrid, Spain
}

A. Cebollada and G. Armelles

Instituto de Microelectrónica de Madrid-IMM (CNM-CSIC), Isaac Newton 8 (PTM) Tres Cantos, 28760 Madrid, Spain

(Received 4 October 2004; accepted 26 February 2005; published online 28 April 2005)

\begin{abstract}
We present a study on the magnetic anisotropy and magnetization reversal of $\mathrm{Fe}(110)$ islands grown on $\mathrm{Al}_{2} \mathrm{O}_{3}$ versus the island size, the island size distribution, and the magnetic interaction between islands. For small islands magnetically connected via a polarizable capping layer, the samples behave as a uniform film with an induced uniaxial in-plane anisotropy. Magnetically isotropic samples are obtained when the islands size is increased. Transverse susceptibility measurements show that the magnetic anisotropy of the particles is macroscopically averaged due to the interisland magnetic interaction. The island size distribution is correlated to the magnetic anisotropy field dispersion. When the capping layer material is $\mathrm{Al}$, a superparamagnetic behavior is found due to the absence of the interisland interaction.
\end{abstract}

(C) 2005 American Institute of Physics. [DOI: 10.1063/1.1895465]

\section{INTRODUCTION}

Research on magnetic nanostructured materials has led to the discovery of physical phenomena which have, in turn, led to technological applications such as ultrahigh-density memory devices and magnetic sensors. These physical phenomena arise from a complex interplay of finite-size effects and surface effects. ${ }^{1}$ The latter effect becomes increasingly important when reducing the particle size because of the increase of the surface atoms to volume atoms ratio, highlighting the importance of surface and interface effects. ${ }^{2}$ Finitesize effects affect those properties that depend on the volume of the particles. One of the most studied finite-size effects in small particle systems is superparamagnetism, since it determines the performance limit of new generation magnetic memory storage media, in which one bit of information has to be written in each single particle. Thermal instability prevents the recording of data since the nanostructures lose their "magnetic memory" in the superparamagnetic regime. To overcome the superparamagnetic limit is one of the greatest objectives in the field of nanostructures for technological applications. The magnetic anisotropy of the particles, as well as the magnitude of the interparticle interactions, plays a key role when it comes to treating the thermal instability of these systems.

In this article, we report the study of the magnetic aniso-

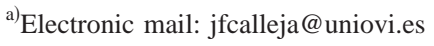

tropy behavior at room temperature of $\mathrm{Fe}(110)$ islands grown on $\mathrm{Al}_{2} \mathrm{O}_{3}$ substrates as a function of the particle size. We also discuss the influence of interparticle interactions. The latter was studied through the modification of the material used as capping.

\section{EXPERIMENTAL PROCEDURE}

Fe was grown at $700{ }^{\circ} \mathrm{C}$ by triode sputtering in order to favor the formation of islands. ${ }^{3}$ In all the samples a $2.5-\mathrm{nm}^{-}$ thick capping layer was grown at room temperature by triode sputtering. The capping layer material was chosen to be a ferromagnetic material $(\mathrm{Fe})$, a polarizable material $(\mathrm{Pt})$, or a nonmagnetic nonpolarizable material $(\mathrm{Al})$. The island size and the physical contact between them were controlled by the deposition time. More details about fabrication, structure, and morphology of the samples can be found elsewhere. ${ }^{3,4}$

The magnetic anisotropy characterization of the samples was carried out by transverse susceptibility (TS) measurements at room temperature. TS measurements performed using a transverse magneto-optical Kerr effect have proved to be a very powerful technique to obtain the magnetic anisotropy field and the anisotropy dispersion in thin films. ${ }^{5-7}$ More recently it has also been applied to obtain an accurate picture of the magnetization processes in epitaxial $\mathrm{Fe} / \mathrm{MgO}$ heterostructures. ${ }^{8}$ TS measurements performed by a magneto-optical Kerr effect basically consist of the application of a small alternating magnetic field $h$ and an orthogonal 
TABLE I. Capping layer used, average island diameter $\left(d_{\mathrm{av}}\right)$, macroscopic magnetic anisotropy field probed by transverse susceptibility measurements $\left(H_{k}\right.$ and $\left.H_{u}\right)$ and coercive field $\left(H_{c}\right)$ of $\mathrm{Fe}(110)$ islands grown on $\mathrm{Al}_{2} \mathrm{O}_{3}$.

\begin{tabular}{ccccc}
\hline \hline Samples & Capping & $d_{\mathrm{av}}(\mathrm{nm})$ & Magnetic anisotropy field $(\mathrm{Oe})$ & $H_{c}(\mathrm{Oe})$ \\
\hline 1 & $\mathrm{Pt}$ & 10 & $H_{u}=30$ & 11 \\
2 & $\mathrm{Pt}$ & 12 & $H_{u}=40$ & 20 \\
3 & $\mathrm{Pt}$ & 14 & $H_{k}=55$ & $37 / 26$ \\
4 & $\mathrm{Pt}$ & 18 & $H_{k}=104$ & $77 / 72$ \\
5 & $\mathrm{Pt}$ & 30 & $H_{k}=210$ & 128 \\
6 & $\mathrm{Fe}$ & 14 & $H_{u}=30$ & 12 \\
7 & $\mathrm{Al}$ & 13 & $\ldots$ & $\cdots$ \\
\hline \hline
\end{tabular}

steady field $H$ both in the film plane. The Kerr signal is proportional to the component of the magnetization parallel to $h(\Delta M)$. If the amplitude of $h$ is small enough, $\Delta M$ is proportional to the susceptibility, and then this magnitude, in a direction parallel to $h$, is measured as a function of $H$. In order to carry out the magnetic characterization of the samples, TS measurements are performed with $H$ applied along different directions as explained in the following paragraphs. The experimental setup is described in Ref. 7. In our case, $h$ had a frequency of $127 \mathrm{~Hz}$. The same experimental setup was used to obtain the hysteresis loops of the samples.

\section{RESULTS AND DISCUSSION}

In the present work we have carried out a systematic study of the transverse susceptibility of Fe nanostructures grown on $\mathrm{Al}_{2} \mathrm{O}_{3}$. The samples' identification, nanostructure size, and the summarized magnetic data are shown in Table I.

Atomic force microscopy (AFM) measurements showed that the samples consisted of islands with rounded tips. The average island diameter is shown in Table I. The samples with smaller islands displayed a homogeneous size distribution. By increasing the island average size, a wider distribution of island sizes was observed. ${ }^{4}$ The insets of Figs. 1(a) and 2 show the AFM images of Pt-coated films with average island diameters of $d=12 \mathrm{~nm}$ and $d=30 \mathrm{~nm}$, respectively. A wider size distribution is displayed in the second case.

In Figs. 1(a) and 1(b) we show the TS vs $H$ curves with $H$ along the hard and easy axes, respectively, for samples with the smallest islands (samples 1 and 2). When transverse susceptibility is measured from $H_{s}$ (saturation field) to $-H_{s}$ through $H=0$, different behaviors are found depending on the measurement direction and on the system under study. In the case of thin films exhibiting a very well-defined macroscopic in-plane uniaxial anisotropy, two characteristic measurement directions are considered: one with $H$ applied along the hard axis of magnetization and another with $H$ applied parallel to the easy axis of magnetization. In the first case two peaks are observed at the uniaxial anisotropy field value in both the positive and the negative field branches, ${ }^{6} H$ $= \pm H_{u}$, and in the second case one peak should be observed at a field value at which the magnetization reverses, i.e., when $H$ equals the coercive field in the negative branch, 5,6 $H=-H_{c}$. As can be seen in Figs. 1(a) and 1(b), samples 1 and 2 present a clear anisotropic behavior. The peaks observed in Fig. 1(a) correspond to the uniaxial induced anisotropy and
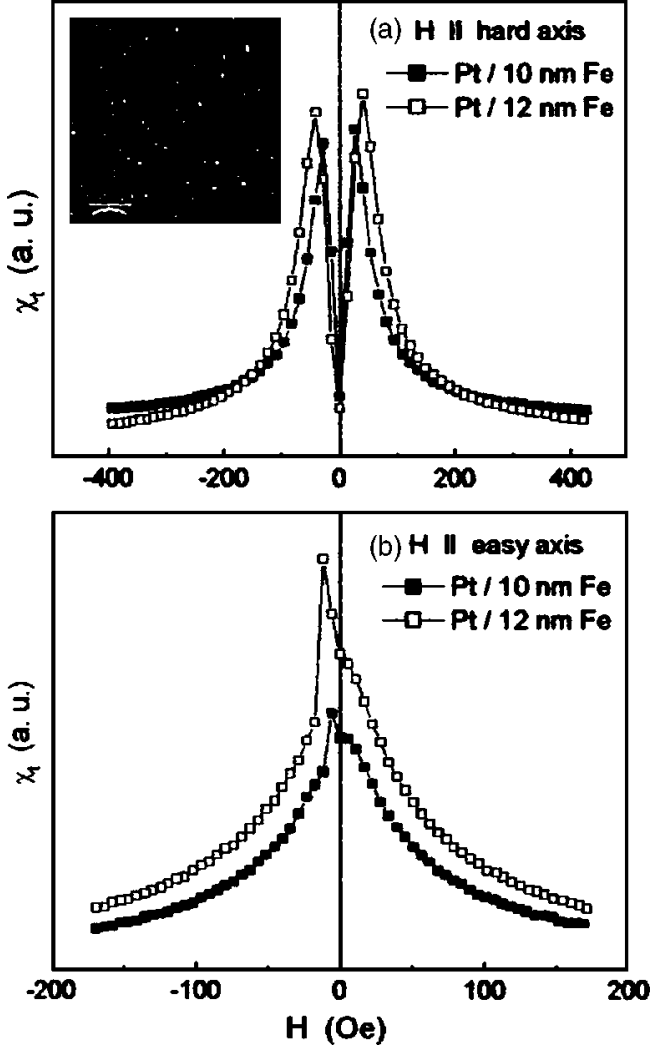

FIG. 1. (a) Transverse susceptibility as a function of $H$, with $H$ along the hard axis for the samples with Pt capping layer and average island diameter $d=10 \mathrm{~nm}$ (solid squares) and $d=12 \mathrm{~nm}$ (open squares). Inset: AFM image of the sample with $d=12 \mathrm{~nm}$. (b) Transverse susceptibility as a function of $H$ with $H$ along the easy axis for the sample with average island diameter $d=10 \mathrm{~nm}$ (solid squares) and $d=12 \mathrm{~nm}$ (open squares).

$H_{u}=30$ Oe for sample 1 and $H_{u}=40$ Oe for sample 2. This small magnetic anisotropy can be growth induced by steps in the substrate ${ }^{9}$ or by oblique incidence. ${ }^{10}$ The peaks observed in Fig. 1(b) correspond to the field at which the magnetization reverses along the macroscopic induced easy axis. The magnetization reversal takes place in the range of 6-11 Oe for sample 1 and 11-17 Oe for sample 2. These values coincide with the $H_{c}$ values estimated from the hysteresis loops (see Table I, only the $H_{c}$ value along the easy axis is given,

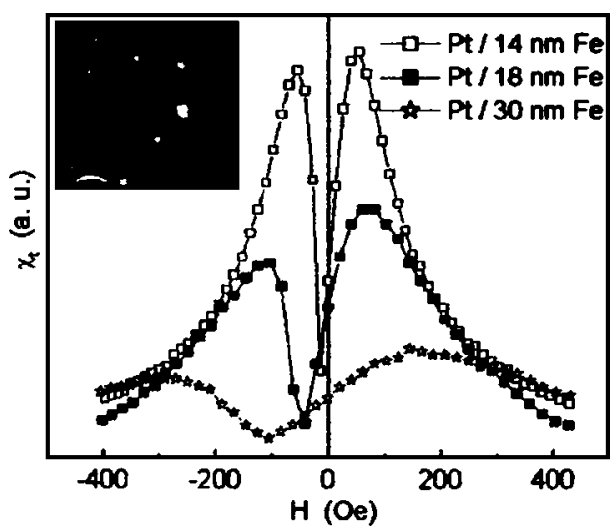

FIG. 2. Transverse susceptibility as a function of $H$ for the samples with $\mathrm{Pt}$ capping layer and average island diameter $d=14 \mathrm{~nm}$ (open squares), $d$ $=18 \mathrm{~nm}$ (closed squares), and $d=30 \mathrm{~nm}$ (open stars). Inset: AFM image of the sample with $d=30 \mathrm{~nm}$. 
$H_{c}$ along the hard axis being null). According to the shape of the TS vs $H$ curves for samples 1 and 2 we conclude that the magnetization reversal along the easy axis takes place mostly by domain-wall displacement, as it takes place in a narrow range of $H$ field values. Since these samples behave like continuous thin films with an in-plane induced uniaxial anisotropy, we can conclude that the $\mathrm{Fe}$ islands are magnetically connected. This is consistent with the fact that Pt becomes polarized in the presence of a ferromagnetic material. ${ }^{11,12}$ In our case, the $\mathrm{Fe}$ islands are magnetically connected via the Pt layer polarization. ${ }^{13}$ In Fig. 2 we display the TS curves for samples with medium and large islands (samples 3, 4, and 5). The shape of the curves was roughly the same independently of the direction along which $H$ was applied. This means that the samples were isotropic. The coercive fields are given in Table I. In the case of samples 3 and 4, the two values given are the maximum and the minimum measured values. They are very similar, which is consistent with the fact that these samples were quasi-isotropic. For sample 5, $H_{c}$ was the same along all measurement directions (the sample was isotropic). In order to understand the TS results, we must consider that in the case of a system of noninteracting randomly oriented single domain particles, each of them with a magnetic anisotropy field $H_{k p}$, three peaks are expected in the TS curves whichever the direction along which $H$ is applied (the system is isotropic): at $H$ $= \pm H_{k p}$ and at $H=-H_{c}$. The peaks observed at $H= \pm H_{k p}$ are due to the contribution of the particles with the easy axis perpendicular to $H$. These peaks have been predicted theoretically ${ }^{14}$ and have been confirmed by experiment. ${ }^{15}$ Three phenomena influence the shape of the experimentally observed curves in these systems: the interparticle magnetic interaction, the anisotropy field value distribution (anisotropy dispersion), and the structure texture (preferred easy axis orientation). The peak in the positive $H$ branch of the curve must be associated with the switch of the magnetization from a direction along $H$ to a direction along the easy axis of these particles. The transition from this direction to a direction along $H$ again produces the peak in the negative $H$ branch of the curve. Three common important features can be observed in the TS curves in Fig. 2: (1) the peaks are observed for $H$ values too low to be identified as $H_{k p}$, (2) the peaks are broadened and the position is not symmetric around $H=0$, and (3) the expected peak at $H=-H_{c}$ is absent. Regarding point (1), if we consider $H_{k p}=2 K_{p} / M_{s}$, taking $M_{s}$ as the saturation magnetization of bulk $\mathrm{Fe}\left(1700 \mathrm{emu} / \mathrm{cm}^{3}\right)$ we obtain $K_{p}=88400 \mathrm{ergs} / \mathrm{cm}^{3}$, a value too low compared to that of the magnetocrystalline anisotropy of bulk Fe. This result can be explained as due to the interparticle magnetic interaction. When studying the magnetic anisotropy of nanostructured materials, which consist of many particles, one must take into account the difference between the macroscopically observed magnetic anisotropy and the intrinsic magnetic anisotropy of one particle. The latter can be even much larger than the corresponding magnetocrystalline anisotropy of the bulk material ${ }^{16}$ since it may contain contributions from shape, strain, and exchange anisotropies. On the other hand, the former should become drastically smaller than the magnetocrystalline anisotropy of one single particle due to interpar- ticle interaction. ${ }^{17,18}$ It should be noted here that TS measurements probe the effective macroscopic anisotropy of the system. Both anisotropies have the same value only in the case of a system of noninteracting particles. Let us call the effective anisotropy field $H_{k}$ in order to distinguish it from the anisotropy field of each particle $H_{k p}$. The peak at $H=$ $-H_{k}$ eventually merges with the peak at $H=-H_{c}{ }^{19}$ Regarding point (2), the anisotropy field dispersion produces a broadening of the peaks at $H= \pm H_{k}$ and an asymmetry of the curve. $^{15,20}$ the peaks are not symmetrically located around $H=0$. In other words, the roundness of the peaks shows that the particles switch over a broad range of $H$ values. Point (3) can be explained if we consider that the peak at $-H_{c}$ can be masked by the peak at $H_{k}$ and taking into account the structure texture. The structure texture affects basically the peak located at $H=-H_{c}$. This peak is more pronounced for a completely random system and it completely vanishes for a system of perfectly oriented particles. ${ }^{14}$ We have roughly estimated the effective magnetic anisotropy field $H_{k}$ of the samples as the mean value of the two observed peaks. The results are listed in Table I. From the results in Table I and the shape of Figs. 1 and 2 we can conclude that when the Fe particle size increases, the samples become isotropic and that both the anisotropy field value and the anisotropy dispersion tend to increase. This is in agreement with the morphological results obtained by AFM. In fact, when the average particle size increases, the island size dispersion increases as well, as we can see in the insets of Figs. 1 and 2.

In order to complete our study, we have analyzed the effect of the material used as capping layer. To do so, we have also carried out transverse susceptibility measurements in samples with $\mathrm{Fe}$ and $\mathrm{Al}$ capping layers. In Fig. 3(a) we show the TS curves for sample 6 (Fe capping layer). The sample is anisotropic, with a uniaxial in-plane induced anisotropy. The solid squares represent the TS for $H$ applied along the easy axis; while the open squares represent TS for $H$ applied along the hard axis. In the inset we show the TS in the first case in the vicinity of $H=0$. As previously discussed in the case of samples 1 and 2, we can see from this figure that the magnetization reverses in a very narrow magneticfield range (between 10 and $11 \mathrm{Oe}$ ), in agreement with the coercive field obtained from the hysteresis loop (see Table I). In this case, the magnetization reversal takes place in a narrower field range than in films 1 and 2. This can be explained if we consider that the Fe islands in sample 6 reverse their magnetization simultaneously. This can be explained if we consider that the $\mathrm{Fe}$ capping layer produces a much stronger magnetic interaction between the islands than the Pt layer, as expected. Again, we can estimate the value of the uniaxial in-plane induced anisotropy from the magnetic-field values at which the peaks in the TS with $H$ along the hard axis are observed [open squares in Fig. 3(a)] as $H_{u}=30$ Oe, a value very similar to those of samples 1 and 2 , which reinforces the hypothesis that the uniaxial induced anisotropy has its origin mainly in the substrate.

The sample with the Al capping was also isotropic, and the transverse susceptibility curve with $H$ applied along any direction resulted, as shown in Fig. 3(b). The most characteristic feature of this curve is that it has only one peak, 


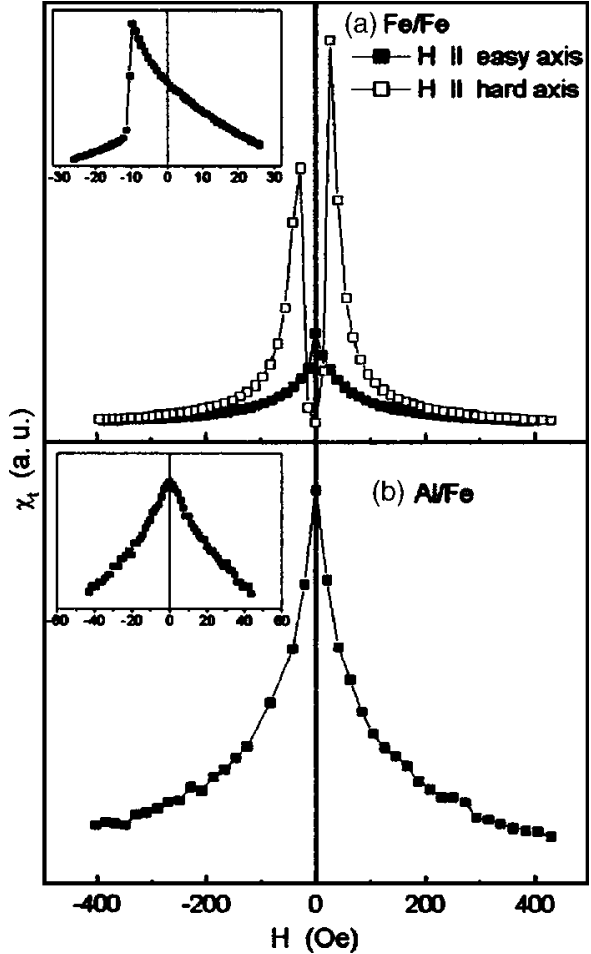

FIG. 3. (a) Transverse susceptibility as a function of $H$ for the sample with Fe capping layer and average island diameter $d=14 \mathrm{~nm}$. The closed squares correspond to $H$ along the easy axis and the open squares to $H$ along the hard axis. Inset: closer view of the transverse susceptibility as a function of $H$, with $H$ along the easy axis in the vicinity of $H=0$. (b) Transverse susceptibility as a function of $H$ for the sample with Al capping layer and average island diameter $d=13 \mathrm{~nm}$. Inset: closer view of the transverse susceptibility as a function of $H$ in the vicinity of $H=0$.

located exactly at $H=0$. The inset of Fig. 3(b) shows in more detail the curve in a narrower $H$ range, in order to show that the peak at $H=0$ is not a scale measurement effect, as no other peaks are observed. Similar results have been obtained by other authors studying the transverse susceptibility of $\mathrm{Fe}$ nanoparticles. $^{21,22}$ On the other hand, the hysteresis loop of this sample had an $\mathrm{S}$ shaped closed loop, with no hysteresis. These results clearly show that this sample is superparamagnetic. A comparison of the TS curves and the results of Table I of samples 3, 6, and 7 help us elucidate the role of the capping layer (see Fig. 4). These three samples have a similar Fe particle diameter. At this point, it is important to know the minimum particle size, which is magnetically stable against thermal demagnetization. The free-energy barrier that the magnetic moment of a particle with anisotropy constant $K_{1}$ must overcome in a switching process is $K_{1} V$, with $V$ as the volume of the particle. The probability per unit time for a particle to switch is given by $P=\nu_{0} \exp \left(-K_{1} V / k_{B} T\right)$, where $\nu_{0}$ is an attempt frequency factor ${ }^{23}$ equal to approximately $10^{9} \mathrm{~s}^{-1}$. Let us consider a spherical Fe particle with an anisotropy energy density equal to that of bulk $\mathrm{Fe}, K_{1}=4.8$ $\times 10^{5} \mathrm{ergs} / \mathrm{cm}^{3}$ at $T=293 \mathrm{~K}$. If we define the stability as a switching probability $P$ of less than $10 \%$ over a specified observation time interval $\tau_{0}$, we can obtain the superparamagnetic radius for stability over the specified time. For example, for 1 year and $1 \mathrm{~s}: r_{0}{ }^{1 \mathrm{yr}} \approx 9 \mathrm{~nm}, r_{0}{ }^{1 \mathrm{~s}} \approx 8 \mathrm{~nm}$. In our case, we can consider our characteristic measurement time as

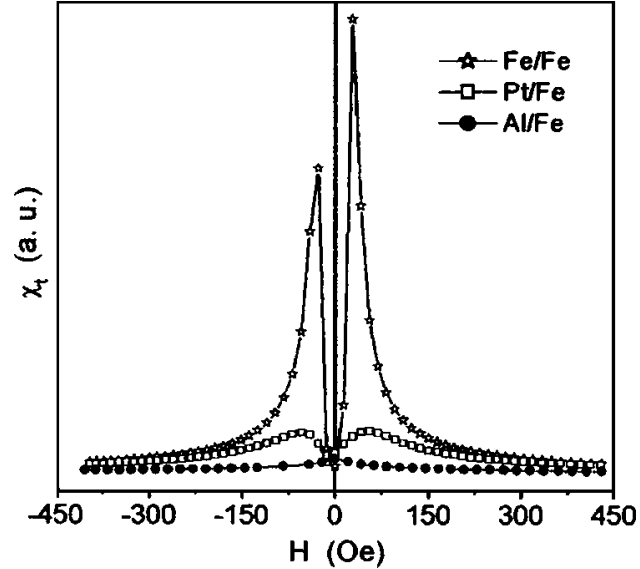

FIG. 4. Transverse susceptibility as a function of $H$ for the sample with Fe capping layer and average island diameter $d=14 \mathrm{~nm}$ with $H$ along the easy axis (open stars), for the sample with Pt capping layer and $d=14 \mathrm{~nm}$, and for the sample with $\mathrm{Al}$ capping layer and $d=13 \mathrm{~nm}$.

the inverse of the frequency of the alternating magnetic field applied $(127 \mathrm{~Hz})$, so $\tau_{0} \approx 8 \mathrm{~ms}$ and $r_{0}{ }^{8 \mathrm{~ms}} \approx 6.6 \mathrm{~nm}$. Samples $1-3,6$, and 7 have Fe particles of mean radius $\leqslant 6-7 \mathrm{~nm}$, so they can have a superparamagnetic behavior at room temperature, as effectively observed in the case of sample 7 . While the particles of samples $1-3$ and 6 are magnetically connected via the capping and so these samples behave as a ferromagnet, the islands in sample 7 must be disconnected in order to explain the superparamagnetic behavior.

\section{CONCLUSIONS}

We have shown that the magnetic anisotropy of Fe islands grown on $\mathrm{Al}_{2} \mathrm{O}_{3}$ depends dramatically on the size of the islands, the distribution of sizes, and the magnetic interaction between the islands. For smaller island size magnetically connected via a capping layer, the samples behave as a continuous film with an induced in-plane uniaxial anisotropy. When the island size increases, the samples become isotropic. The transverse susceptibility measurements in these systems would allow the determination of the magnetic anisotropy of each island. However, the magnetic anisotropy probed macroscopically is averaged due to the magnetic interaction among the islands.

When a nonpolarizable capping layer is used ( $\mathrm{Al})$ a superparamagnetic behavior is observed for samples that show a ferromagnetic behavior with either a magnetic $(\mathrm{Fe})$ or a polarizable $(\mathrm{Pt})$ capping layer. This can be used as a hint to avoid superparamagnetism in nanostructured Fe.

\section{ACKNOWLEDGMENTS}

The Spanish Commission of Science and Technology is acknowledged for financial support. One of the authors (Y.H.) thanks the "Ramón y Cajal" program.

${ }^{1}$ X. Batlle and A. Labarta, J. Phys. D 35, R15 (2002).

${ }^{2}$ P. Grünberg, J. Phys.: Condens. Matter 13, 769 (2001).

${ }^{3}$ C. Quintana, J. L. Menéndez, Y. Huttel, M. Lancin, E. Navarro, and A. Cebollada, Thin Solid Films 434, 228 (2003).

${ }^{4}$ E. Navarro, Y. Huttel, C. Clavero, A. Cebollada, and G. Armelles, Phys. Rev. B 69, 224419 (2004). 
${ }^{5}$ E. Feldtkeller, Z. Physik 176, 510 (1963).

${ }^{6}$ H. Hoffmann, Phys. Status Solidi 33, 175 (1969).

${ }^{7}$ M. C. Contreras, J. F. Calleja, M. Rivas, M. O. Gutiérrez, and J. A. Corrales, J. Magn. Magn. Mater. 175, 64 (1997).

${ }^{8}$ C. Martínez Boubeta, A. Cebollada, J. F. Calleja, C. Contreras, F. Peiró, and A. Cornet, J. Appl. Phys. 93, 2126 (2003).

${ }^{9}$ J. L. Menendez, Ph.D. thesis, University of Oviedo, 2001.

${ }^{10}$ Y. Park, E. E. Fullerton, and S. D. Bader, Appl. Phys. Lett. 66, 2140 (1995).

${ }^{11}$ R. Bertaco and F. Ciccaci, Phys. Rev. B 57, 96 (1998).

${ }^{12}$ W. J. Antel, Jr., M. M. Schwickert, and T. Lin, Phys. Rev. B 60, 12933 (1999).

${ }^{13}$ E. Navarro, Y. Huttel, C. Clavero, A. Cebollada, and G. Armelles, Appl. Phys. Lett. 84, 2139 (2004).

${ }^{14}$ A. Hoare, R. W. Chantrell, W. Schmitt, and A. Eiling, J. Phys. D 26, 461 (1993).
${ }^{15}$ L. Paretti and G. Turilli, J. Appl. Phys. 61, 5098 (1987).

${ }^{16}$ Nanomaterials: Synthesis, Properties and Applications, edited by A. S. Edelstein (Institute of Physics, Bristol, 2002).

${ }^{17}$ I. A. Bardsley and V. S. Speriosu, IEEE Trans. Magn. 26, 2718 (1990).

${ }^{18}$ T. Shimatsu, M. Takahashi, M. Suekane, M. Miyamura, K. Yamaguchi, and H. Yamasaki, Proceedings of the Third IUMRS International Conference on Advanced Materials (1993) (unpublished).

${ }^{19}$ Ch. Chang and J. Yang, Appl. Phys. Lett. 65, 496 (1994).

${ }^{20}$ P. Poddar, J. L. Wilson, H. Srikanth, D. F. Farrell, and S. A. Majetich, Phys. Rev. B 68, 214409 (2003).

${ }^{21}$ L. Spinu, H. Srikanth, J. A. Wiemann, S. Li, J. Tang, and C. J. O'Connor, IEEE Trans. Magn. 36, 3032 (2000).

${ }^{22}$ Y. Shiratuchi, M. Yamamoto, Y. Endo, D. Li, and S. D. Bader, J. Appl. Phys. 94, 7675 (2003).

${ }^{23}$ R. C. O'Handley, Modern Magnetic Materials: Principles and Applications (Wiley, New York, 2000). 